\title{
Type 2 diabetes compromises the value of non-invasively measured augmentation index in predicting the severity of coronary artery disease: a hospital-based observational study
}

\author{
Sijing Wu, Yujie Zhou*, Yueping Li, Yuyang Liu, Dongmei Shi, Xiaoli Liu, Wei Liu, Yi Yu and Shuo Jia
}

\begin{abstract}
Background: Central hemodynamic indices have been demonstrated to correlate with coronary artery disease (CAD). However, in the context of type 2 diabetes mellitus (DM), this correlation has not been fully illustrated. Therefore, this study was employed to investigate the impact of DM on the correlation between aortic augmentation index and the severity of coronary artery disease.

Methods: In this study, we analyzed 197 patients who underwent coronary angiography at Anzhen Hospital from September 2015 to January 2016. Central hemodynamics were non-invasively measured with BPro ${ }^{\circledR}$ device (Health STATS, Singapore). The severity of CAD was defined according to SYNTAX scores. Type 2 diabetes was defined according to ADA guidelines. Alx@75 was defined as Alx normalized to a heart rate of 75 bpm. Receiver operating characteristics (ROC) determined the optimal cut-off value of Alx@75 to predict moderate to severe CAD. Multivariate logistic regression analysis evaluated the correlation between central hemodynamic parameters and CAD severity.

Results: Eighty-four (42.6\%) of the studied subjects were diabetic patients. Our findings were that (1) Alx@75 was significantly correlated with SYNTAX. (ROC analyzed AUC: $0.638,95 \% \mathrm{Cl} 0.555-0.721, p<0.05$ ). The cut-off value of Alx@75 to predict moderate-to-severe CAD as SYNTAX score more than 22 was 71.45. (2) In non-diabetic patients, correlation analysis revealed that AIx@75, augmentation pressure and peak relative time were significantly correlated with CAD severity $(p<0.05)$. After adjustment, Alx@75 remained as the only independent predictor of moderate-tosevere CAD (odds ratio 1.099, $95 \% \mathrm{Cl} 1.028-1.176, p<0.05$ ). (3) In diabetic patients, the correlation between central hemodynamic parameters and the severity of CAD did not exist.
\end{abstract}

Conclusions: Aortic augmentation index was significantly related to the severity of CAD and was an independent predictor of severe CAD. However, clinical practitioners should note that its value in DM populations was compromised.

Keywords: Coronary artery disease, Type 2 diabetes mellitus, Augmentation index, SYNTAX score

* Correspondence: azzyj12@163.com

Beijing Anzhen Hospital, Capital Medical University, Beijing Institute of Heart

Lung and Blood Vessel Disease, Beijing, China

(c) The Author(s). 2016 Open Access This article is distributed under the terms of the Creative Commons Attribution 4.0 International License (http://creativecommons.org/licenses/by/4.0/, which permits unrestricted use, distribution, and reproduction in any medium, provided you give appropriate credit to the original author(s) and the source, provide a link to the Creative Commons license, and indicate if changes were made. The Creative Commons Public Domain Dedication waiver (http://creativecommons.org/publicdomain/zero/1.0/) applies to the data made available in this article, unless otherwise stated. 


\section{Background}

Central pressures are revealed to be better related to cardiovascular events than are peripheral pressures [1]. Furthermore, central pressures are pathologically more relevant than peripheral pressures with CAD [2]. The advancement of non-invasive techniques to measure central blood pressure has allowed for safer and wider application in more patients. Augmentation pressure (AP), aortic augmentation index (AIx) and pulse wave analysis-derived peak relative time (PRT) are markers of arterial stiffness [3]. Recently, non-invasively measured central hemodynamic parameters have been reported to be related with CAD $[4,5]$. However, no reliable cut-off value for such parameters has been determined.

Type 2 diabetes mellitus (DM) is a common comorbidity for CAD. Diabetic patients with CAD demonstrate accelerated progression of coronary atherosclerosis, leading to worse clinical outcome [6]. Arterial stiffness can increase in the presence of overt DM. Central hemodynamics is significantly altered in diabetic patients [3, 7]. Yet whether the coexistence of DM exerts any influence on the relationship between central hemodynamic parameters and CAD remains unclear.

Previous studies have failed to elucidate the effect of comorbidities like type 2 diabetes mellitus on the correlation between central hemodynamic indices and CAD. In view of these considerations, we initiated the present study to investigate the correlation between non-invasively measured central hemodynamic parameters and the severity of CAD. Meanwhile, we explored in detail the influence of type 2 diabetes on the correlation.

\section{Methods}

\section{Study design and patient recruitment}

The cross-sectional study was performed at Anzhen Hospital in Beijing. We consecutively included patients who underwent coronary angiography at our center from September 2015 to January 2016. Exclusion criteria: 1. Patients without complete baseline data; 2. Patients whose central pressure measurement was not readable; 3 . Patients who had previous coronary intervention or coronary artery bypass grafting, atrial fibrillation, or hemodynamically significant valvular heart disease were excluded. This study was approved by the Institutional Ethics Committee of Anzhen Hospital and all subjects gave written informed consent.

\section{Measurement of blood pressure and pulse wave analysis} Noninvasive blood pressure and pulse wave analysis were performed prior to coronary angiography with the commercially available $\mathrm{BPro}^{\circ}$ device with A-Pulse CASP software (Health STATS, Singapore). The BPro ${ }^{\circ}$ device captured the radial waveform, which was subsequently used to generate central systolic pressure by $\mathrm{N}$-point moving average method as described before [8]. Peripheral pressure waveforms were recorded from the wrist using applanation tonometry. After 20 sequential waveforms had been obtained, augmentation pressure (AP), augmentation index (AIx) and peak relative time (PRT) were derived from the measurement of PWA. The central hemodynamic indices are illustrated in Fig. 1. AP was defined as the difference between P1 and P2, AIx was calculated as AP divided by pulse pressure and PRT was described as T2T1 duration. Given that AIx is inversely related to heart rate, we used AIx normalized to a heart rate of $75 \mathrm{bpm}$ (AIx@75) as an alternative. The $\mathrm{BPro}^{\circ}$ device measured arterial pressure waveforms in 10s blocks over the course of 20-30s and the first stable waveform block was used for data analysis. The experienced operator visually inspected the waveforms for anomalies and selected the first stable waveform block, which applied to the criteria of adequate pulse height $(100 \mathrm{mV})$ and pulse length variability $(<20 \%)$ [9]. Each patient was measured three times to avoid bias.

\section{Analysis of coronary angiography}

Coronary angiography (CAG) was primarily performed through the trans-radial approach with standardized technique. To reach consensus, all coronary angiograms were visually assessed by at least two experienced interventional cardiologists. The extent and severity of coronary artery disease were determined by SYNTAX scores. The anatomical SYNTAX score was calculated with online calculator version 2.1 at www.syntaxscore.com. The following is 12categorization of CAD severity assessed by SYNTAX score according to guidelines: low as $\leq 22$, moderate as $23-32$ and severe as $\geq 33[10,11]$.

\section{Statistical analysis}

The data were analyzed with the SPSS 15.0 software (Chicago, IL, USA). Continuous variables were presented as mean \pm standard deviation (SD) and categorical variables as percentages. Comparisons between groups were made by using the unpaired student $t$ test (two-tailed) or Mann-Whitney U-test for continuous data and the Pearson's $X^{2}$ - or Fisher's exact test. Spearman's correlation coefficient (two-tailed) was used for the analysis of the correlation between AIx and SYNTAX scores. The receiver-operating characteristic curve was used to evaluate the predictive accuracy of AIx@75 for moderate to severe CAD; the highest Youden index ( $J$ statistic) representing the maximum potential effectiveness was used to determine the optimal cut-off AIx@75 value. Logistic regression analysis was employed for multivariate and univariate analysis. A p value of $<0.05$ was considered statistically significant. 


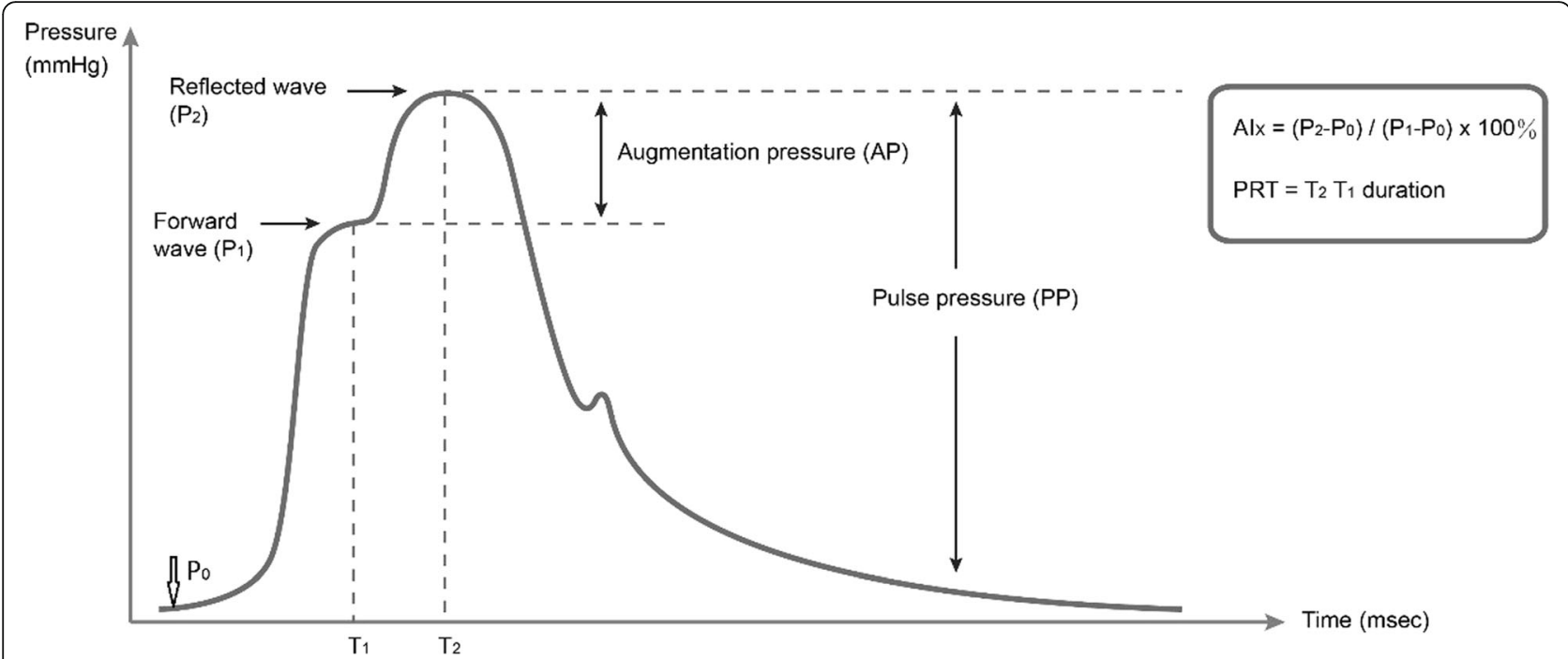

Fig. 1 Pressure waveform measurement. Alx = augmentation index PRT = peak relative time

\section{Results}

\section{Patient demographics}

Our study consecutively enrolled 318 patients. A total of 85, 20 and 16 patients were excluded due to a history of percutaneous coronary intervention, coronary bypass grafting and atrial fibrillation, respectively. The analysis of 197 participants compared 113 non-diabetic with 84 diabetic patients. Baseline characteristics are summarized in Table 1. The mean age of subjects was $59.2 \pm$ 11.1 years old, and $72.1 \%$ were men. The distribution of

Table 1 Baseline characteristics

\begin{tabular}{|c|c|c|c|c|}
\hline & $\begin{array}{l}\text { Total } \\
(n=197)\end{array}$ & $\begin{array}{l}\text { Non-diabetic } \\
(n=113)\end{array}$ & $\begin{array}{l}\text { Diabetic } \\
(n=84)\end{array}$ & $p$-value \\
\hline Age(years) & $59.2 \pm 11.1$ & $57.9 \pm 11.0$ & $60.9 \pm 11.0$ & 0.069 \\
\hline Gender(male), n(\%) & $142(72.1)$ & $82(72.6)$ & $59(70.2)$ & 0.646 \\
\hline Hypertension, n(\%) & $128(65)$ & $67(59.3)$ & $61(72.6)$ & 0.063 \\
\hline Hyperlipidemia, n(\%) & $45(22.8)$ & 21(18.6) & $24(28.6)$ & 0.106 \\
\hline Current smokers, n(\%) & $101(51.3)$ & $59(52.2)$ & $41(48.8)$ & 0.592 \\
\hline $\operatorname{BMI}\left(\mathrm{kg} / \mathrm{m}^{2}\right)$ & $25.2 \pm 5.3$ & $25.4 \pm 4.9$ & $25.1 \pm 5.7$ & 0.684 \\
\hline SYNTAX & $16.4 \pm 12.2$ & $16.0 \pm 11.9$ & $17.0 \pm 12.6$ & 0.546 \\
\hline SYNTAX > 22, n(\%) & $60(30.5)$ & $35(31.3)$ & 25(29.8) & 0.823 \\
\hline \multicolumn{5}{|l|}{ Lab data } \\
\hline $\mathrm{HbA} 1 \mathrm{c}(\%)$ & $6.4 \pm 1.2$ & $5.7 \pm 0.4$ & $7.3 \pm 1.3$ & $<0.3^{*}$ \\
\hline Triglyceride(mmol/l) & $1.8 \pm 1.6$ & $1.6 \pm 0.8$ & $2.0 \pm 1.8$ & 0.073 \\
\hline LDL cholesterol(mmol/l) & $2.6 \pm 1.0$ & $2.6 \pm 1.0$ & $2.5 \pm 1.0$ & 0.775 \\
\hline HDL cholesterol(mmol/l) & $1.1 \pm 0.3$ & $1.1 \pm 0.3$ & $1.1 \pm 0.3$ & $0.045^{*}$ \\
\hline Creatinine $(\mu \mathrm{mol} / \mathrm{l})$ & $79.0 \pm 17.6$ & $77.8 \pm 17.5$ & $80.6 \pm 17.7$ & $0.260^{*}$ \\
\hline \multicolumn{5}{|l|}{ Medications } \\
\hline ACEI, n(\%) & $21(10.7)$ & $11(9.7)$ & 10(11.9) & 0.641 \\
\hline ARB, $n(\%)$ & 17(8.6) & $6(5.3)$ & $11(13.1)$ & 0.057 \\
\hline$\beta$-blockers, n(\%) & $35(17.8)$ & $21(18.6)$ & $14(16.7)$ & 0.706 \\
\hline Calcium channel blockers, n(\%) & $44(22.3)$ & $14(12.4)$ & $30(35.7)$ & $<0.3^{*}$ \\
\hline Statins, $n(\%)$ & $32(16.2)$ & 10(8.8) & $22(26.2)$ & $0.9^{*}$ \\
\hline
\end{tabular}

${ }^{*} p$ value for comparison between the non-diabetic and diabetic groups; $p<0.05$ indicates statistical significance. $B M I$ body mass index, $L D L$ low-density lipoprotein, $H D L$ high-density lipoprotein, $A C E l$ angiotensin converting enzyme inhibitor, $A R B$ angiotensin receptor blocker 
hypertension, dyslipidemia and current smoking status were not significantly different between groups $(p>0.05)$, nor were laboratory findings and medications.

\section{Central hemodynamic indices analysis}

Table 2 shows the values of hemodynamic indices. Mean values of central aortic systolic pressure (CASP), central pulse pressure (central PP) and brachial pulse pressure (brachial PP) were significantly higher in the diabetic group $(p<0.05)$.

In the total patient group, univariate analysis revealed that AIx@75 was significantly correlated with SYNTAX score. AIx@75 value to predict the presence of moderateto-severe CAD was 71.45 (ROC defined AUC 0.638; sensitivity $75 \%$, specificity $47 \%$, $95 \%$ CI $0.555-0.721, p=0.4)$.

In addition, the correlation between central hemodynamic indices and SYNTAX score differed between groups: univariate logistic analysis showed that higher AIx@75, AP and lower PRT were significantly correlated with SYNTAX score in the non-diabetic group, but not correlated in the diabetic group. Similarly, multivariate logistic analysis showed that higher AIx@75 was significantly correlated with SYNTAX score in the non-diabetic group only (Table 3). Figure 2 demonstrates the linear correlation between AIx@75 and SYNTAX score categorized as non-diabetic $\left(R^{2}=0.180, p<0.3\right)$ and diabetic groups $\left(R^{2}=0.4, p=0.680\right)$.

\section{Discussion}

To the best of our knowledge, this is the first study investigating the impact of type 2 diabetes on the relationship between non-invasively measured central hemodynamic parameters and CAD.

Table 2 Hemodynamic indices

\begin{tabular}{lcccc}
\hline & $\begin{array}{l}\text { Total } \\
(n=197)\end{array}$ & $\begin{array}{l}\text { Non-diabetic } \\
(n=113)\end{array}$ & $\begin{array}{l}\text { Diabetic } \\
(n=84)\end{array}$ & $p$-value \\
\hline SBP(mmHg) & $130.1 \pm 17.3$ & $127.8 \pm 17.5$ & $133.2 \pm 16.6$ & $0.037^{*}$ \\
DBP(mmHg) & $74.9 \pm 11.1$ & $74.8 \pm 10.9$ & $75.0 \pm 11.5$ & 0.884 \\
CASP(mmHg) & $120.3 \pm 16.6$ & $118.1 \pm 16.5$ & $123.1 \pm 16.5$ & $0.044^{*}$ \\
MAP(mmHg) & $91.9 \pm 12.1$ & $91.3 \pm 12.8$ & $92.8 \pm 11.2$ & 0.387 \\
AP(mmHg) & $11.8 \pm 7.5$ & $11.9 \pm 7.3$ & $11.7 \pm 7.9$ & 0.840 \\
Central PP(mmHg) & $45.4 \pm 13.0$ & $43.5 \pm 11.9$ & $48.1 \pm 13.9$ & 0.130 \\
Brachial PP(mmHg) & $55.2 \pm 14.0$ & $53.2 \pm 13.2$ & $58.1 \pm 14.5$ & 0.130 \\
PR(bpm) & $67.7 \pm 10.1$ & $67.5 \pm 10.3$ & $67.8 \pm 9.8$ & 0.750 \\
Alx(\%) & $77.8 \pm 14.5$ & $77.0 \pm 14.0$ & $79.0 \pm 15.0$ & 0.372 \\
Alx@75(\%) & $74.9 \pm 14.2$ & $74.0 \pm 14.3$ & $76.1 \pm 13.9$ & 0.317 \\
PRT(ms) & $114.5 \pm 27.0$ & $116.1 \pm 28.1$ & $112.5 \pm 25.4$ & 0.374 \\
\hline
\end{tabular}

${ }^{*} p$ value for comparison between the non-diabetic and diabetic groups; $p<0.05$ indicates statistical significance. SBP systolic blood pressure, DBP diastolic blood pressure, MAP mean arterial pressure, $P P$ pulse pressure, $P R$ pulse rate, $C A S P$ central aortic systolic pressure, $A P$ augmentation pressure, $P R T$ peak relative time

\section{Augmentation index and coronary artery disease}

Cho SW et al. found that AIx was significantly associated with the extent of CAD in the younger group [5]. Tanindi A et al. demonstrated that AIx was correlated with more severe CAD despite patients' clinical presentation [12]. Similarly, we confirmed that AIx was positively correlated with SYNTAX. Moreover, AIx is an independent predictor of moderate-to-severe CAD. It has been well demonstrated that augmentation index is a parameter reflecting arterial stiffness. However, an established theory explaining why the severity of atherosclerosis in CAD relates to parameters of arterial stiffness is lacking. The concept of bidirectional influence is a possible explanation, namely elevated parameters of arterial stiffness are both a cause and a consequence of atherosclerosis $[5,13]$. On the one hand, increased central BP pulsation promotes endothelial damage, leading to the progress of atherosclerosis; On the other hand, diffused atherosclerotic plaques impair the elastic properties of the arterial wall. Physiologically, the reflect wave arrives earlier in the aorta and augments pressure in late systole as aortic stiffness increases. Augmentation of the aortic pressure wave is a manifestation of wave reflection, which can be expressed as the AP [5]. A noteworthy study focused on the time duration analysis and unveiled that augmentation time ratio was related to the presence of CAD [4]. Combined with our finding of the relationship between peak relative time and the severity of CAD, the theory that advanced atherosclerosis significantly increases wave reflection is reasonable.

\section{The role of diabetes mellitus}

We categorized our patients into diabetic versus nondiabetic groups and found that the predictive value of AIx was profound in non-diabetic patients only. Our findings are in line with Agnoletti D et al. [14] who found no specific role of AIx in DM. However, JH Chow et al. [15] recruited both diabetic and non-diabetic patients and revealed that higher AIx@75 was universally associated with PCI among CAD patients. Moreover, MT Schram et al. [16] found that DM was associated with increased AIx. No definitive conclusion has been made regarding the impact of DM on AIx in the CAD population.

AIx is defined as a ratio of augmentation pressure and pulse pressure. Therefore, it is necessary to consider both elements when analyzing its changes. It has been proposed that global stiffening of arteries augments systolic pressure mainly through the increase of wave reflections, whereas local stiffening of proximal aorta amplifies forward pressure wave $[4,17]$. Studies concerning arterial pathology in diabetic subjects discovered that DM may lead to preferential increase of large artery stiffness, resulting in lower reflection magnitude [3]. Based on such potential mechanisms and our findings, we suggest that diabetic patients may experience more prominent stiffening of the proximal 
Table 3 Odds ratios for the association between the central hemodynamic parameters and the risk of more severe CAD measured by SYNTAX

\begin{tabular}{|c|c|c|c|c|c|c|}
\hline \multirow[t]{2}{*}{ Variables } & \multirow[t]{2}{*}{ N } & \multirow{2}{*}{$\begin{array}{l}\text { SYNTAX > } \\
22, N(\%)\end{array}$} & \multicolumn{2}{|l|}{ Univariate analysis } & \multicolumn{2}{|l|}{ Multivariate analysis $^{a}$} \\
\hline & & & Odds ratio(95 \% Cl) & $p$ & Odds ratio(95 \% Cl) & $p$ \\
\hline Total & 197 & $60(30.5)$ & & & & \\
\hline Alx@75 & & & $1.035(1.011-1.059)$ & 0.004 & & \\
\hline AP & & & $1.031(0.989-1.075)$ & 0.145 & & \\
\hline PRT & & & $0.991(0.980-1.002)$ & 0.120 & & \\
\hline Non-Diabetic & 113 & $35(31.3)$ & & & & \\
\hline Alx@75 & & & $1.064(1.027-1.103)$ & 0.001 & 1.099(1.028-1.176) & 0.006 \\
\hline $\mathrm{AP}$ & & & $1.084(1.018-1.154)$ & 0.012 & 0.938(0.837-1.051) & 0.273 \\
\hline PRT & & & $0.985(0.971-1.000)$ & 0.044 & $1.002(0.984-1.021)$ & 0.800 \\
\hline Diabetic & 84 & $25(29.8)$ & & & & \\
\hline Alx@75 & & & $1.002(0.969-1.037)$ & 0.885 & & \\
\hline AP & & & $0.979(0.921-1.040)$ & 0.491 & & \\
\hline PRT & & & $1.001(0.983-1.020)$ & 0.920 & & \\
\hline
\end{tabular}

andices that were statistically significant in the univariate logistic regression analysis were included in the multivariate analysis

aorta compared with their non-diabetic peers. As a consequence, diabetes may blunt the correlation between AIx and atherosclerosis-induced arterial stiffness in CAD.

Type 2 diabetes has been proven to be associated with increased central artery stiffness $[14,16,18]$. In a large population-based study, JA Chirinos et al. [19] concluded that DM was associated with increased aortic stiffening, but not with increased carotid stiffness. Subjects with DM demonstrated a decreased reflection magnitude. Prolonged exposure to hyperglycemia can lead to protein glycation, collagen crosslinking and endothelial dysfunction [20]. All of the abnormal changes have deleterious effects on arterial stiffness. Possibly, as DM progresses, these modifications can increase aortic stiffness by a local effect on the arterial wall despite classical determinants of arterial stiffness [14]. Accordingly, AIx may not be the optimal estimation of arterial stiffness and predictor of the severity of CAD in the diabetic population. Future investigations should evaluate the predictive value of AIx in distinct clinical scenarios. As for diabetic CAD patients, experimental research of pathological changes to the aorta is warranted. Previous studies have found that in DM patients, PP was an independent predictor of CAD [19]. Future studies may identify other meaningful predictive indices (such as PP) for diabetic CAD patients. Moreover, central hemodynamic parameters are presumably affected by patient conditions such as impaired renal function [21], so a comprehensive study including all central

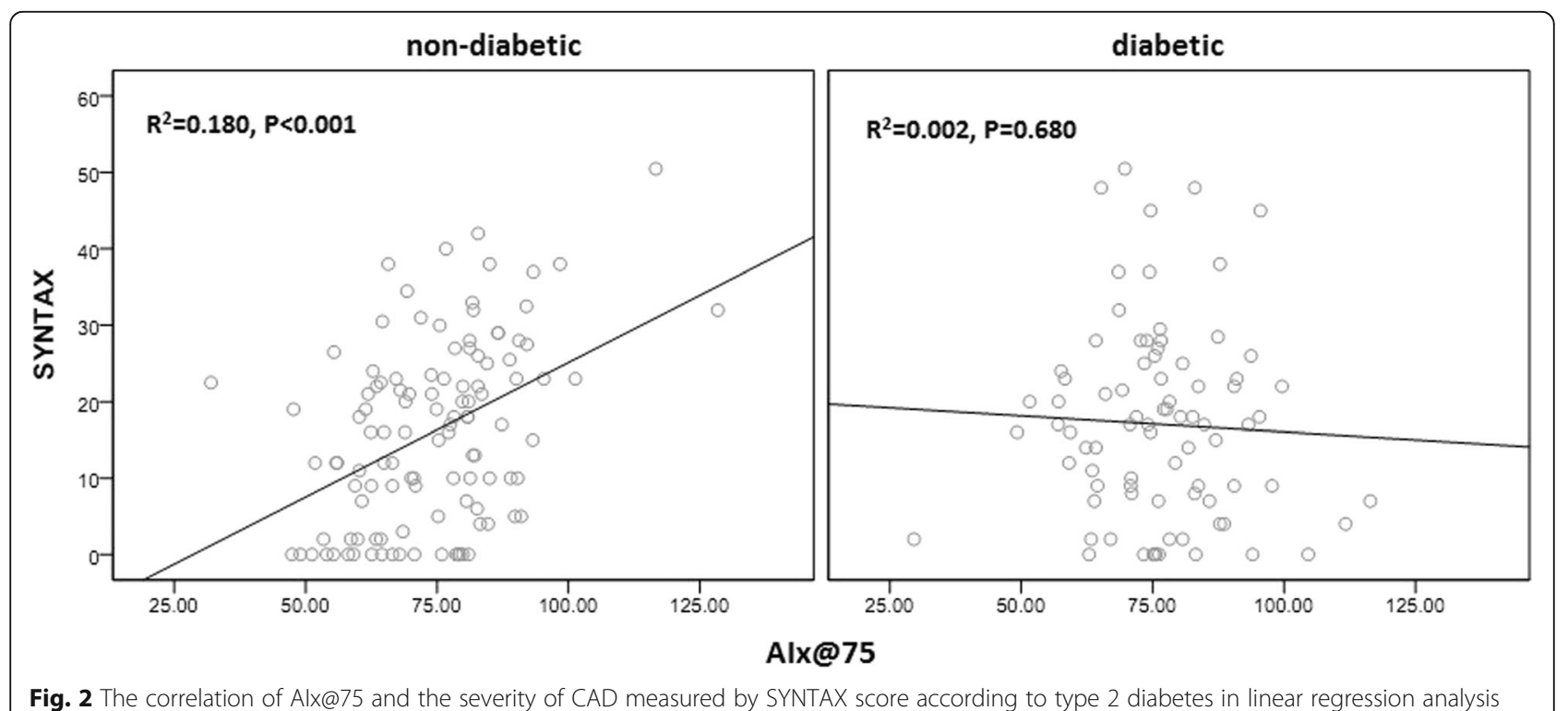

Fig. 2 The correlation of Alx@75 and the severity of CAD measured by SYNTAX score according to type 2 diabetes in linear regression analysis 
hemodynamic status-related factors might be valuable in the future.

\section{Limitations}

First, the present study was limited by its relatively small sample size; thus, future research evaluating a larger number of patients is necessary. Second, the study's observational design, permitted potential confounders to affect the result even after adjustment. Third, long-term follow up studies should be implemented to explore the possible predictive value of AIx for cardiovascular outcomes in non-diabetic patients. Forth, the current study didn't record patient-reported duration of DM and DMrelated organ damage. It will be valuable to investigate the impact of the duration or status of the disease on the correlation in future research.

\section{Conclusions}

Aortic augmentation index is significantly related to the severity of CAD and is an independent predictor of more severe CAD. However, its value in DM population was compromised, which might be related to DMspecific arterial changes and clinical practitioners should be aware of the fact.

\section{Abbreviations}

Alx: Augmentation index; Alx@75: Augmentation index normalized to heart rate of 75 bpm; AP: Augmentation pressure; CAD: Coronary artery disease; CASP: Central aortic systolic pressure; DM: Type 2 diabetes mellitus; PRT: Peak relative time

\section{Acknowledgements}

We hereby thank and acknowledge the assistance of Dr. Panpan Hu in the statistical analysis and precious advice of this work.

\section{Funding}

This work was supported by the Beijing Municipal Administration of Hospitals Clinical Medicine Development of Special Funding Support (code: ZYLX2903) and the National Key Clinical Specialty Construction Project (29-213) for study design, the "Beijing Municipal Administration of Hospitals" Ascent Plan (Code: DFL2170601) for data collection and analysis.

\section{Availability of data and materials}

The data of this study will not be shared publically because they will be applied for further researches of this series. But authors do agree that the data can be shared individually if requested.

\section{Authors' contributions}

SW, WL, YY participated in the design of the study and performed the statistical analysis. $Y L, Y L$ and DS conceived of the study, and participated in its design and coordination and helped to draft the manuscript. $Y Z, X L$ and SJ performed intervention and measurement of patients and acquisition of funding. All authors read and approved the final manuscript.

\section{Competing interests}

The authors declare that they have no competing interests.

\section{Consent for publication}

Not applicable.

\section{Ethics approval and consent to participate}

The present study was approved by the Institutional Ethics Committee of Anzhen Hospital. Informed consent was obtained from all participants.
Received: 7 April 2016 Accepted: 5 November 2016

Published online: 10 November 2016

\section{References}

1. McEniery CM, Cockcroft JR, Roman MJ, et al. Central blood pressure: current evidence and clinical importance. Eur Heart J. 2014;35(26):1719-25.

2. Agabiti-Rosei $E$, Mancia G, O'Rourke MF, et al. Central blood pressure measurements and antihypertensive therapy: a consensus document. Hypertension. 2007;50(1):154-60.

3. Prenner SB, Chirinos JA. Arterial stiffness in diabetes mellitus. Atherosclerosis 2015:238(2):370-9.

4. Mizuno A, Miyauchi K, Nishizaki Y, et al. Impact of the augmentation time ratio on direct measurement of central aortic pressure in the presence of coronary artery disease. Hypertens Res. 2015;38(10):684-9.

5. Cho SW, Kim BK, Kim JH, et al. Non-invasively measured aortic wave reflection and pulse pressure amplification are related to the severity of coronary artery disease. J Cardiol. 2013;62(2):131-7.

6. Kataoka Y, Shao M, Wolski K, et al. Multiple risk factor intervention and progression of coronary atherosclerosis in patients with type 2 diabetes mellitus. Eur J Prev Cardiol. 2013:20(2):209-17.

7. Chen JY, Chou CH, Lee YL, et al. Association of central aortic pressures indexes with development of diabetes mellitus in essential hypertension. Am J Hypertens. 2010;23(10):1069-73.

8. Williams B, Lacy PS, Yan P, et al. Development and validation of a novel method to derive central aortic systolic pressure from the radial pressure waveform using an n-point moving average method. J Am Coll Cardiol. 2011;57(8):951-61.

9. Lacy PS, Brunel P, Baschiera F, et al. Effects of exercise on central aortic pressure before and after treatment with renin-angiotensin system blockade in patients with hypertension. J Renin Angiotensin Aldosterone Syst. 2015; 16(4):1052-60.

10. Amsterdam EA, Wenger NK, Brindis RG, et al. 2014 AHA/ACC guideline for the management of patients with non-ST-elevation acute coronary syndromes: executive summary: a report of the American College of Cardiology/American Heart Association Task Force on Practice Guidelines. Circulation. 2014;130(25):2354-94.

11. Windecker $\mathrm{S}$, Kolh P, Alfonso F, et al. 2014 ESC/EACTS Guidelines on myocardial revascularization: The Task Force on Myocardial Revascularization of the European Society of Cardiology (ESC) and the European Association for Cardio-Thoracic Surgery (EACTS)Developed with the special contribution of the European Association of Percutaneous Cardiovascular Interventions (EAPCI). Eur Heart J. 2014;35(37):2541-619.

12. Tanindi A, Erkan AF, Alhan A, et al. Central pulse pressure amplification is associated with more extensive and severe coronary artery disease. Scand CardiovasC J. 2014:48(3):167-75

13. Safar ME, Blacher J, Jankowski P. Arterial stiffness, pulse pressure, and cardiovascular disease-is it possible to break the vicious cycle? Atherosclerosis. 2011;218(2):263-71.

14. Agnoletti $D$, Lieber $A$, Zhang $Y$, et al. Central hemodynamic modifications in diabetes mellitus. Atherosclerosis. 2013:230(2):315-21.

15. Choi J, Kim SY, Joo SJ, et al. Augmentation index is associated with coronary revascularization in patients with high Framingham risk scores: a hospital-based observational study. BMC Cardiovasc Disord. 2015;15:131.

16. Schram MT, Henry RM, van Dijk RA, et al. Increased central artery stiffness in impaired glucose metabolism and type 2 diabetes: the Hoorn Study. Hypertension. 2004;43(2):176-81.

17. Reymond P, Westerhof N, Stergiopulos N. Systolic hypertension mechanisms: effect of global and local proximal aorta stiffening on pulse pressure. Ann Biomed Eng. 2012;40(3):742-9.

18. Zhang M, Bai Y, Ye P, et al. Type 2 diabetes is associated with increased pulse wave velocity measured at different sites of the arterial system but not augmentation index in a Chinese population. Clin Cardiol. 2011;34(10):622-7.

19. Chirinos JA, Segers P, Gillebert TC, et al. Central pulse pressure and its hemodynamic determinants in middle-aged adults with impaired fasting glucose and diabetes: the Asklepios study. Diabetes Care. 2013;36(8):2359-65.

20. Stehouwer CD, Henry RM, Ferreira I. Arterial stiffness in diabetes and the metabolic syndrome: a pathway to cardiovascular disease. Diabetologia. 2008:51(4):527-39.

21. Tebaldi M, Biscaglia S, Fineschi $M$, et al. Fractional flow reserve evaluation and chronic kidney disease: analysis from a multicenter Italian registry (the FREAK study). Catheter Cardiovasc Interv. 2015. doi:10.1002/ccd.26364. 